\title{
TBH score: a new model to predict and prevent severe liver damage after chemotherapy for cancer patients
}

This article was published in the following Dove Press journal: Cancer Management and Research

\author{
Mengping Zhang, ${ }^{\text {, } *}$ \\ Yong Bao, 2,* Wei Chen, ${ }^{3, *}$ \\ Mengchao Wei, ${ }^{4}$ Hui Pang, ${ }^{5}$ \\ Yu Feng Ren, ${ }^{2}$ Jie Mei, ${ }^{6}$ \\ Sheng Ye, 'Shunjun Fu, ${ }^{7}$ \\ Zhen Wei Peng ${ }^{1,6}$ \\ 'Department of Oncology, The First \\ Affiliated Hospital, Sun Yat-sen University, \\ Guangzhou, China; ${ }^{2}$ Department of \\ Radiation Oncology, The First Affiliated \\ Hospital, Sun Yat-sen University, Guangzhou, \\ China; ${ }^{3}$ Department of Pancreatobiliary \\ Surgery, The First Affiliated Hospital, Sun \\ Yat-sen University, Guangzhou, China; \\ ${ }^{4}$ Department of Liver Surgery, The First \\ Affiliated Hospital, Sun Yat-sen University, \\ Guangzhou, China; ${ }^{5}$ Department of Medical \\ Records Management, The First Affiliated \\ Hospital, Sun Yat-sen University, Guangzhou, \\ China; ${ }^{6}$ Clinical Trials Unit, The First \\ Affiliated Hospital, Sun Yat-sen University, \\ Guangzhou, China; ${ }^{7}$ Department of \\ Hepatobiliary Surgery, Zhujiang Hospital, \\ Southern Medical University, Guangzhou, \\ China
}

*These authors contributed equally to this work

Correspondence: Zhen Wei Peng Department of Oncology and the Clinical Trials Unit, The First Affiliated Hospital, Sun Yat-sen University, 58 Zhongshan Road 2, Guangzhou 510080, China Tel +862087 7557668576

Email pzhenw@mail.sysu.edu.cn

Shunjun Fu

Department of Hepatobiliary Surgery, Zhujiang Hospital, Southern Medical University, Middle Industrial Avenue 253\#, Guangzhou 510280, China Email fsj103@I63.com
Purpose: To explore a quantitative predictive model for the risk of chemotherapy-induced severe liver damage (CISLD).

Materials and methods: In total, 3870 consecutive cancer patients initially treated with chemotherapy were retrospectively collected and randomly assigned to a training $(n=2580)$ or internal validation $(n=1290)$ set in a $2: 1$ ratio to construct and validate the model. Additional external validation was performed using another data set $(n=413)$. A total of 486 patients were prospectively enrolled to assess the clinical significance of the model. CISLD was defined as grade $\geq 3$ hepatotoxicity.

Results: CISLD was found in 255 (9.9\%), 128 (9.9\%) and $36(8.7 \%)$ patients in the training, internal and external validation sets, respectively. Serum triglyceride, body mass index and history of hypertension formed the basis of the score model. Patients could be stratified into low, intermediate and high-risk groups with $<10 \%, 10-30 \%$ and $>30 \%$ CISLD occurrence, respectively. This model displayed a concordance index (C-index) of 0.834 and was validated in both the internal (C-index, 0.830) and external (C-index, 0.817) sets. The incidence of CISLD was significantly reduced in those who received preventive hepatoprotective drugs compared to those who did not among patients assessed as the intermediate risk group (8.9\% vs $17.5 \%, p=0.042)$ and the high risk group $(15.6 \%$ vs $55.8 \%, p=0.043)$.

Conclusions: The new score model can be used to accurately predict the risk of CISLD in cancer patients undergoing chemotherapy. Clinically, this can be translated into a reference tool for oncologists in the clinical decision-making process before chemotherapy to provide appropriate prevention and interventions for patients with a high risk of CISLD.

Keywords: chemotherapy, liver damage, predictive model

\section{Background}

All chemotherapeutic agents, whether used in monotherapy or combination therapy, can directly or indirectly cause liver toxicity due to hepatocellular injury, inflammation and/or cholestasis. Since most hepatotoxic drug reactions are idiosyncratic and can mechanistically be classified as immune (hypersensitive) or metabolic, ${ }^{1}$ these reactions are usually unpredictable. Moreover, the degree of adverse liver reactions is heterogeneous due to the differences in susceptibility to drug-induced liver injury or capacity to recover from injury between individual patients. The clinical manifestations of chemotherapy-related hepatotoxicity can vary from asymptomatic transient liver biochemical abnormalities to acute diseases with jaundice. ${ }^{2}$ Severe liver damage can lead to a dose reduction of the chemotherapeutic agents, treatment delays or 
interruptions, or even fatal adverse events. Dose reductions or course delays may reduce the effect of anti-tumor therapy, ${ }^{3}$ and fatal consequences are worthy of attention. ${ }^{4,5}$

Previous literature and studies have reported several factors, including body mass index (BMI), age, sex, tobacco and alcohol intake, and treatment cycles, that may increase the risk of liver damage after chemotherapy. ${ }^{6-11}$ In addition to the above factors, chemotherapy-induced liver toxicity is often idiosyncratic and may also be affected by genetic factors, comorbidities, cancer subtypes and other factors. ${ }^{12}$ Oncologists have already realized that the above factors are associated with potential severe liver damage after chemotherapy. However, thus far, not all individual factors have been combined to establish a quantitative predictive model for the risk of chemotherapy-induced severe liver damage (CISLD) that aims to guide the treatment choice before chemotherapy.

Therefore, we conducted a study to identify the highrisk factors for predicting CISLD and to develop a reliable prediction model by collecting as much comprehensive clinical data and laboratory data as possible from a large sample of patients. The model can be used as a reference tool for oncologists in the clinical decision-making process when selecting treatment agents and determining the dosages before chemotherapy.

\section{Methods}

\section{Patients and design}

The study retrospectively analyzed a total of 3870 patients diagnosed with primary cancer from January 1, 2010 to December 31, 2017 in the First Affiliated Hospital, Sun Yat-sen University. Patient consent to review their medical records was obtained from all patients entered in the study and supported by the Institutional Ethics Committee of the First Affiliated Hospital, Sun Yat-sen University. The inclusion criteria were as follows: (1) 18 years or older; (2) confirmed cancer by histopathological examination; and (3) first treatment was chemotherapy and finished at least one cycle of chemotherapy. The exclusion criteria were as follows: (1) previous anticancer therapies; (2) missing necessary clinical information and laboratory data; and (3) no follow-up data. (4) known history of chronic liver disease. We randomly allocated these patients into a training cohort and an internal validation cohort in a 2:1 ratio. In addition, we included another 413 cancer patients with the same criteria from January 1, 2015 to December 31, 2016 from the Cancer Center, Sun Yat-sen
University, as the external validation cohort. The study was censored on January 31, 2018. The study was approved by the Institutional Ethics Committee of the First Affiliated Hospital, Sun Yat-sen University. CISLD was defined when the grade or indices reflecting hepatic function was over 3, according to the Common Terminology Criteria for Adverse Events (CTCAE) after chemotherapy. ${ }^{13}$ The liver functions is checked once a week during treatment and per one-three months during follow up. The time frames of hepatotoxicity was defined as any time in the future after chemotherapy, and the doctors judged whether the hepatotoxicity was related to the chemotherapy.

\section{Data collection}

The relevant data were retrieved from the medical records at the time of diagnosis and during the follow-up period. We collected the following background data: age; BMI; sex; history of smoking and alcoholism; history of hypertension, coronary heart disease (CHD) and diabetes; hepatitis B surface antigen (HBsAg); hepatitis C virus (HCV); nucleotide analogue medication; laboratory data (albumin [ALB], blood urea nitrogen [BUN], creatinine [Cr], uric acid [UA], blood glucose [GLU], alanine aminotransferase [ALT], aspartate aminotransferase [AST], ratio of alanine aminotransferase to aspartate aminotransferase [ALT/ AST], cholesterol [CHOL], triglyceride [TG], C-reactive protein [CRP], cystatin $\mathrm{C}$ [Cys], hemoglobin [Hb], total bilirubin [TBIL], gamma-glutamyl transpeptidase [GGT], activated partial thromboplastin time [APTT], fibrinogen [Fbg], international normalized ratio [INR]); tumor type; tumor staging; liver metastasis; cycle of chemotherapy; and chemotherapeutics (oxaliplatin, cisplatin, carboplatin, irinotecan, gemcitabine, fluorouracil, paclitaxel, docetaxel, pemetrexed, doxorubicin).

\section{Statistical analysis}

Statistical analysis was conducted using R studio software (version 1.0.153, https://www.rstudio.com/products/rstu dio/). The $\mathrm{R}$ packages used in the study include the glmnet, Hmisc and rms packages. Continuous variables are presented as the mean \pm standard deviation, while categorical variables are presented as the frequencies and percentages of events. Continuous variables were compared using Student's $t$-test or Kruskal-Wallis H test (variables with an abnormal distribution), and categorical variables were compared using the $\chi^{2}$ test or Fisher's exact test. The least absolute shrinkage and selection 
operator (LASSO) method was used to primarily identify features for predicting hepatic function damage in the training cohort. Then, the significant variables identified by LASSO were incorporated into further multivariate logistic regression analysis to build the final prediction model. The nomogram was constructed based on the results of the multivariate logistic regression analysis following LASSO. The predictive performance of the nomogram was evaluated by the concordance index (C-index) and calibration curve. The $\mathrm{C}$-index was an equivalent variable of the area under the curve (AUC) of the receiver operating characteristic (ROC) curve for censored data. A calibration curve was applied to assess the agreement between the nomogram-predicted result and the actual observed result. In addition, internal and external validation was performed by applying the derived nomogram in the validation cohorts. The $\mathrm{C}$-index and calibration curve were also used in the validation cohorts. All statistical tests were two-sided with a statistical significance level set at $p<0.05$. Moreover, the regression coefficients of the significant variables were multiplied by 10 and rounded to acquire point numbers facilitating the bedside calculation of a risk score. The risk score was utilized to assign patients into different risk groups.

\section{Results}

\section{Patient characteristics and outcomes}

The patient characteristics of the training and internal validation cohorts are listed in Table 1. In total, 3870 patients diagnosed with cancer during the study period were enrolled in this study. Among these patients, 2580 were assigned to the training cohort, while the other 1290 were assigned to the internal validation cohort. As shown in Table 1, the training and internal validation cohorts showed good agreement in all variables. In total, 276 patients received a nucleotide analogue for hepatitis B infection; among whom, 188 (7.9\%) were in the training cohort, and $88(6.8 \%)$ were in the validation cohort. The patient characteristics of the external cohort are listed in Table S1. CISLD was found in 255 (9.9\%), 128 $(9.9 \%)$ and $36(8.7 \%)$ patients in the training, internal and external validation cohorts, respectively. The median time of developing severe hepatic function damage from the first dose of chemotherapy in the three sets of patients was $27 \mathrm{~d}$, $30 \mathrm{~d}$, and $29 \mathrm{~d}$, respectively. The details of hepatic function damage are listed in Tables S2 and S3.

Feature selection using LASSO and multivariate logistic regression analysis was performed in the training cohort. After LASSO analysis, 42 features were reduced to 3 potential predictors based on the 2870 patients in the training cohort. Figures $\mathrm{S} 1$ and $\mathrm{S} 2$ show the process of selecting features using LASSO. The optimized lambda $(\lambda)$ determined in Figure S1 was utilized to identify features with nonzero coefficients from the coefficient profile plot in Figure S2. LASSO identified TG, BMI and history of hypertension as potential prognostic variables that predict CISLD. After incorporating the three variables into the multivariate logistic regression analysis, TG (hazard ratio $[\mathrm{HR}], 2.269 ; 95 \%$ confidence interval [CI], $1.937-2.661 ; p<0.001)$, BMI (HR, 10.274; 95\% CI, 7.047-15.476; $p<0.001)$ and history of hypertension (hazard ratio [HR], 2.028; 95\% confidence interval [CI], 1.407-2.895; $p<0.001)$ showed statistical significance. The details of the LASSO method and multivariate analysis are shown in Table 2.

\section{Development of the prognostic nomogram in the training cohort}

The final three variables were incorporated into the nomogram to predict CISLD in patients after chemotherapy (Figure 1). The nomogram included two background characteristics (BMI and history of hypertension) and one laboratory test (TG). Every value of the variables was assigned a specific score. To use the nomogram, an individual patient's value is located on the axis of each variable, and a line is drawn upwards to determine the number of points assigned to each variable value. The sum of these numbers is located on the total point axis, and a line is drawn downwards to the risk axis to determine the likelihood of hepatic function damage.

\section{Predictive performance of the prognostic nomogram in the training and internal/ external validation cohorts}

The C-index of the nomogram for predicting CISLD was 0.834 (95\% CI: $0.815-0.854)$, which is greater than 0.7 , suggesting that the new model has advanced suitability. The calibration plot demonstrated good agreement between the actual observations and the nomogram predictions for the risk of hepatic function damage in the training cohort (Figure 2). In the internal validation cohort, the nomogram displayed a C-index of 0.830 (95\% CI: 0.803-0.857) for estimating the risk of hepatic function damage. Good calibration was also observed in the internal validation cohort (Figure 3 ). In the external validation 


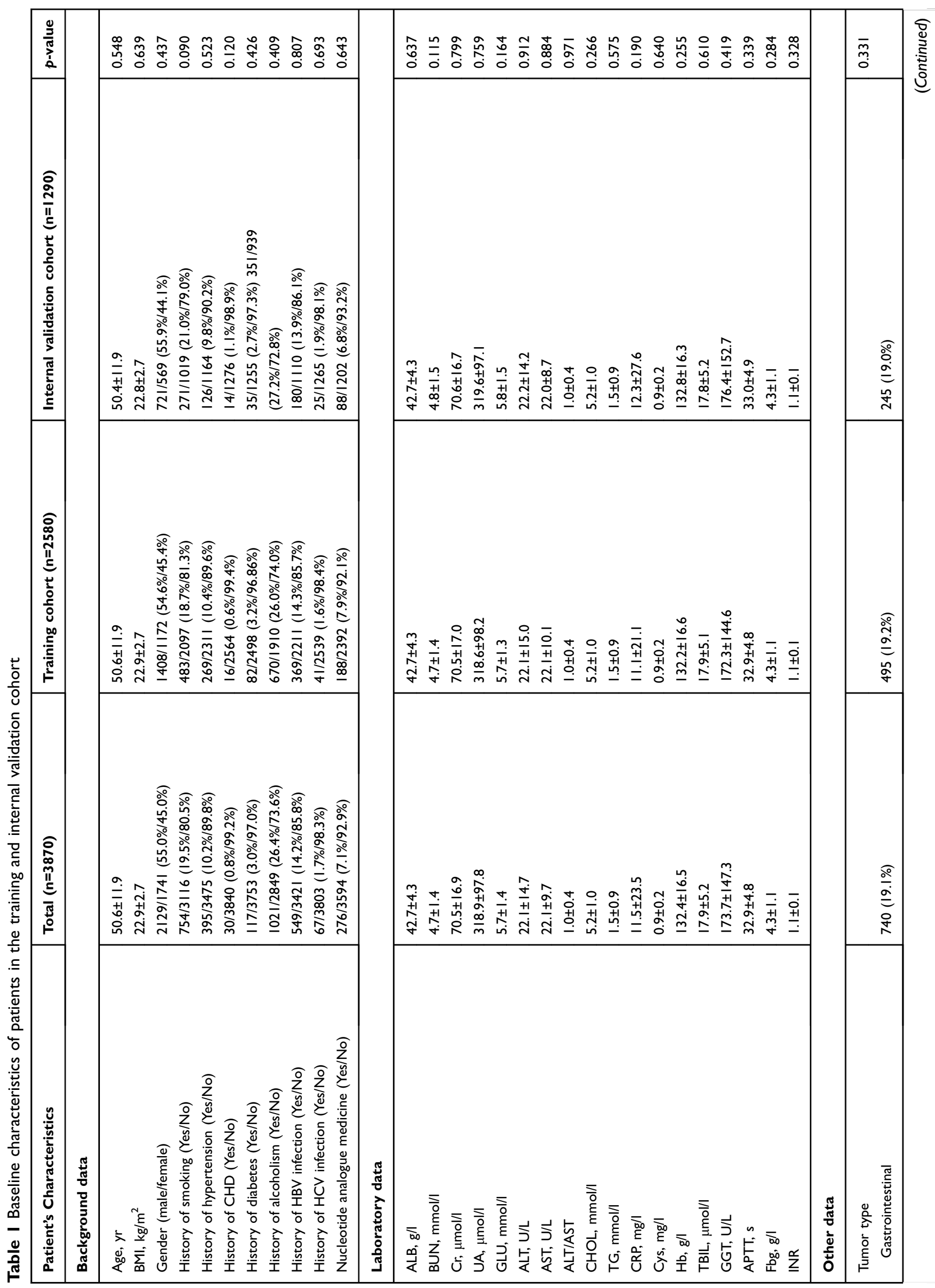




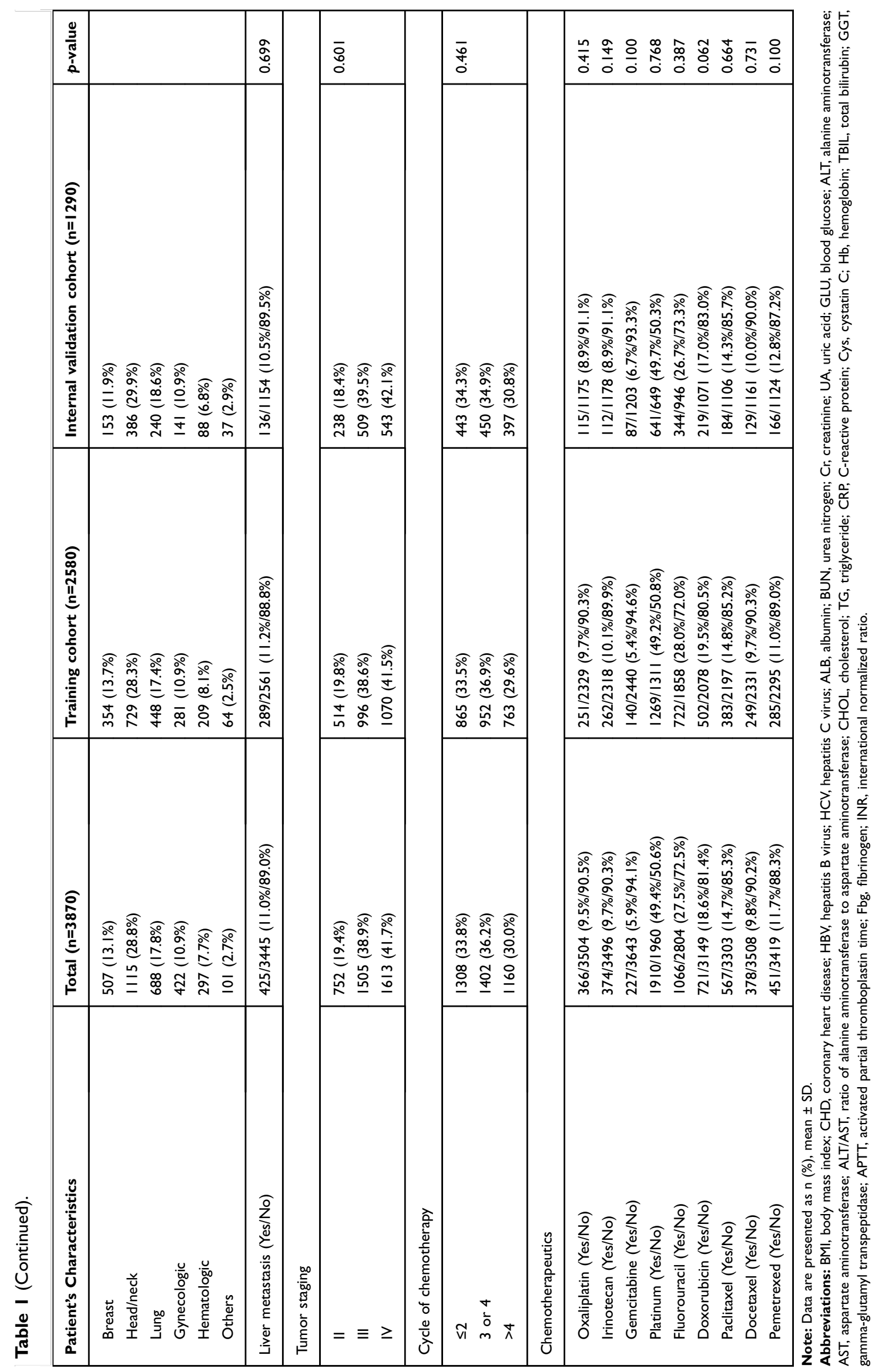


Table 2 LASSO-multivariate logistic regression results of predicting hepatic function damage after chemotherapy in training cohort

\begin{tabular}{|l|l|l|l|}
\hline & \multicolumn{3}{|l|}{$\begin{array}{l}\text { LASSO-multivariate logistic } \\
\text { regression }\end{array}$} \\
\hline Variables & $\beta$ & $p$-value & Hazard ratio $(95 \% \mathrm{Cl})$ \\
TG & $0.8 I 9$ & $<0.00 I^{*}$ & $2.269(\mathrm{I} .937,2.66 \mathrm{I})$ \\
BMI & 2.330 & $<0.00 I^{*}$ & $10.274(7.047,15.476)$ \\
History of hypertension & $0.34 \mathrm{I}$ & $<0.00 I^{*}$ & $2.028(\mathrm{I} .407,2.895)$ \\
\hline
\end{tabular}

Note: *Statistically significant at alpha $=0.05$

Abbreviations: TG, triglyceride; BMI, body mass index.

cohort, the nomogram displayed a C-index of 0.817 (95\% CI: 0.752-0.882) for estimating the risk of hepatic function damage. Good calibration was also observed in the external validation cohort (Figure S3).

\section{TBH score calculation to identify different risk groups}

The calculated regression coefficients were multiplied 10 times and rounded to facilitate the calculation of a risk score (TBH, TG-BMI-history of Hypertension score) (Table 3). We then calculated the TBH score for patients in the training cohort $(n=2870)$ and identified three subgroups with distinct outcomes. Patients whose TBH score was $\leq 16$ points $(1488 / 2580,57.7 \%)$ had a rate of
CISLD $<10 \%$, which was significantly different from that of patients with a TBH score of 16 to $\leq 39$ points (861/2580, 33.3\%), who had a rate of CISLD of 10-30\%. In contrast, patients with a TBH score $>39$ points $(231 / 2580,9.0 \%)$ had a rate of CISLD $>30 \%$. Since the average rate in the whole population is approximately $9.9 \%$, we defined patients with $\mathrm{TBH}$ scores $\leq 16$ as the low-risk group, 16 to $\leq 39$ points as the moderate-risk group and $>39$ points as the high-risk group. Crucially, the TBH score was validated in the internal $(n=1290)$ and external $(n=413)$ validation cohorts. Among the 1290 patients in the internal validation cohort, the number of patients with TBH scores $\leq 16$ points, 16 to $\leq 39$ points, $>39$ points were 759 (58.8\%), $433(33.6 \%)$ and $98(7.6 \%)$, respectively. The corresponding possibility of CISLD for the patients in each group was $<10 \%, 10-30 \%$, and $>30 \%$, respectively. Among the 413 patients in the external validation cohort, the number of patients with TBH scores $\leq 16$ points, 16 to $\leq 39$ points, $>39$ points were $261(63.2 \%), 140$ $(33.9 \%)$ and $12(2.9 \%)$, respectively. The corresponding possibility of CISLD for the patients in each group was $<10 \%, 10-30 \%,>30 \%$, respectively. The percentage of CISLD and 95\% Confident Interval of the low, intermediate and high-risk group for internal and external datasets were showed in Table 4.
Points

TG

BMI

History.of.hypertension

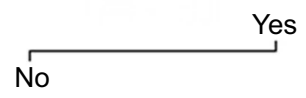

Total Points

Yes

(n)

No

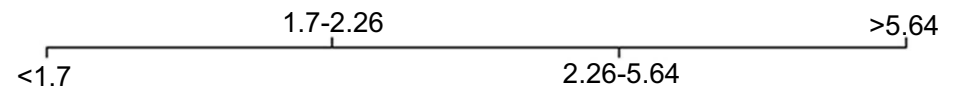

$\geq 23$

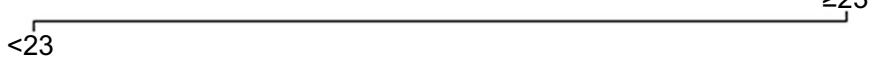

$\begin{array}{lllllllllllll}0 & 20 & 40 & 60 & 80 & 100 & 120 & 140 & 160 & 180 & 200 & 220 & 240\end{array}$

Risk

Figure I Nomogram for predicting hepatic function damage in patients after chemotherapy. Abbreviations: TG, triglyceride; BMI, body mass index. 


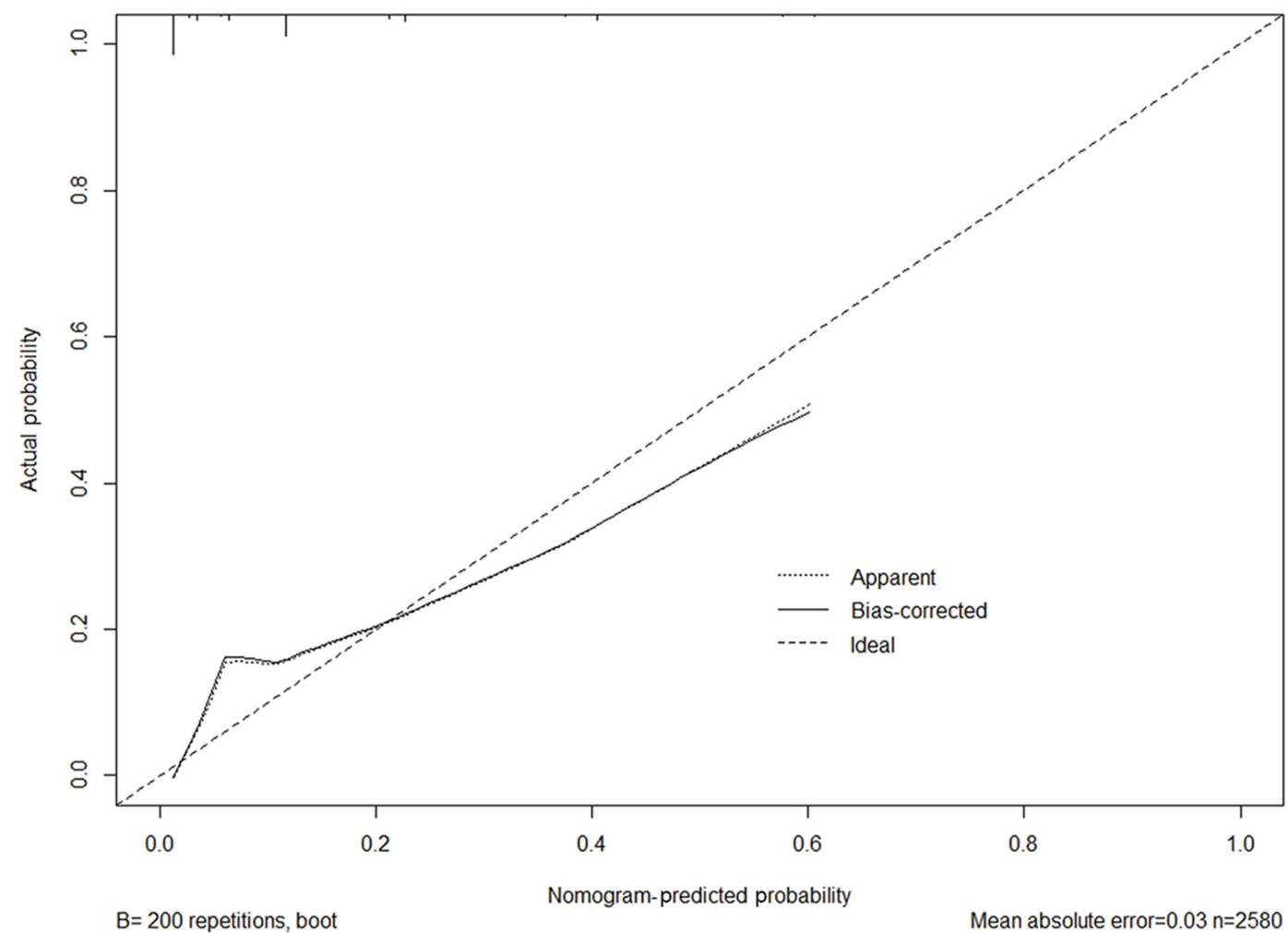

Figure 2 The calibration plot for the risk of hepatic function damage in the training cohort.

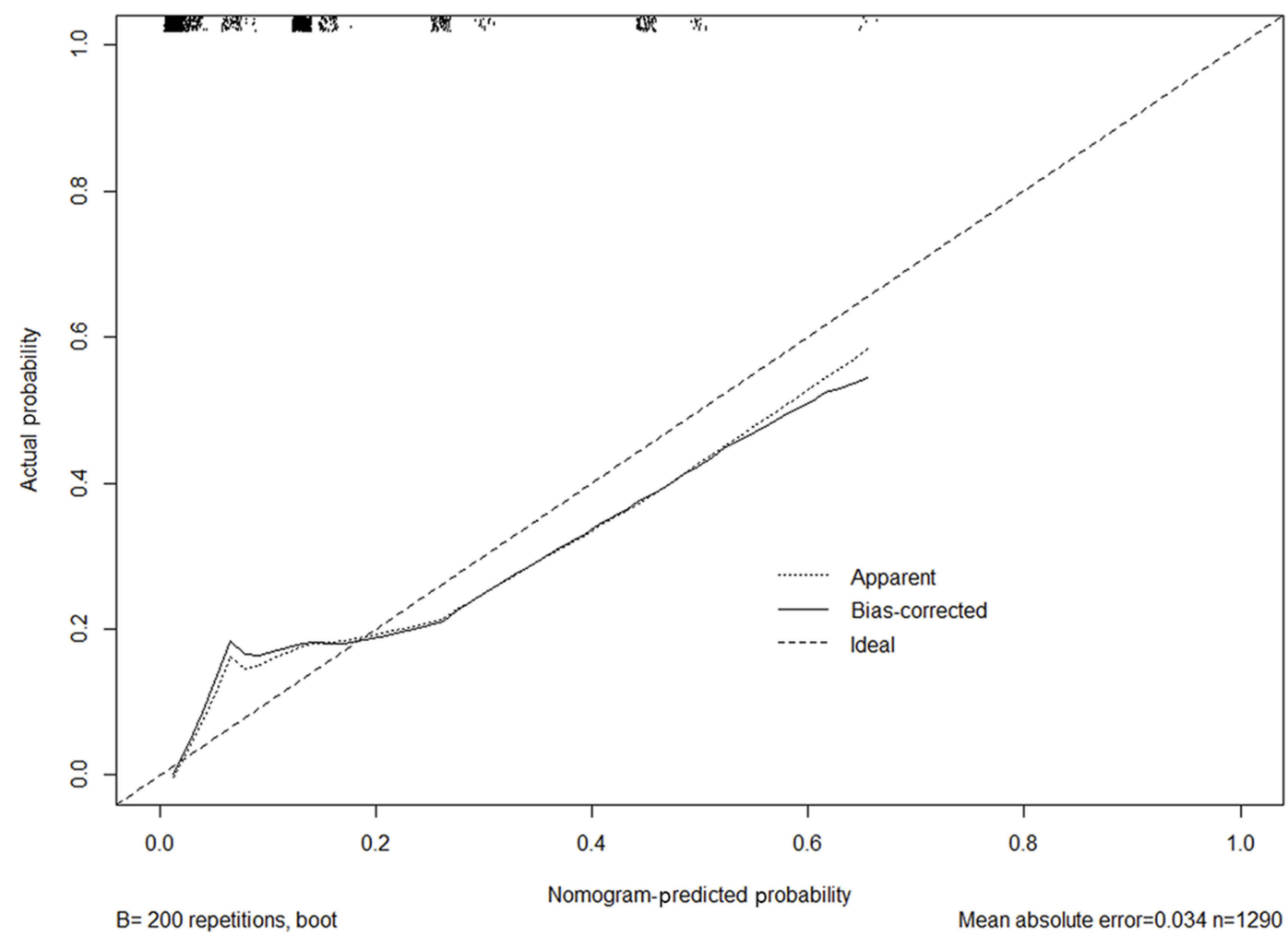

Figure 3 The calibration plot for the risk of hepatic function damage in the internal validation cohort. 
Table 3 Stratification of significant variables used to calculate bedside TBH (TG-BMI-history of Hypertension) score

\begin{tabular}{|c|c|c|c|c|}
\hline Variables & Stratification & $\boldsymbol{\beta}$ & TBH score points & $p$-value* \\
\hline $\begin{array}{l}\text { TG } \\
(\mathrm{mmol} / \mathrm{l})\end{array}$ & $\begin{array}{l}<1.7 \\
1.7-2.26 \\
2.26-5.64 \\
\geq 5.64\end{array}$ & $\begin{array}{l}- \\
0.423 \\
1.576 \\
3.248\end{array}$ & $\begin{array}{l}0 \\
4 \\
16 \\
32\end{array}$ & $\begin{array}{l}0.03 I^{*} \\
<0.00 I^{*} \\
<0.00 I^{*}\end{array}$ \\
\hline $\begin{array}{l}\text { BMI } \\
\left(\mathrm{kg} / \mathrm{m}^{2}\right)\end{array}$ & $\begin{array}{l}<23 \\
\geq 23\end{array}$ & $\begin{array}{l}- \\
2.371\end{array}$ & $\begin{array}{l}0 \\
24\end{array}$ & $<0.00 I^{*}$ \\
\hline History of hypertension & $\begin{array}{l}\text { No } \\
\text { Yes }\end{array}$ & $\begin{array}{l}- \\
0.732\end{array}$ & $\begin{array}{l}0 \\
7\end{array}$ & $<0.00 I^{*}$ \\
\hline
\end{tabular}

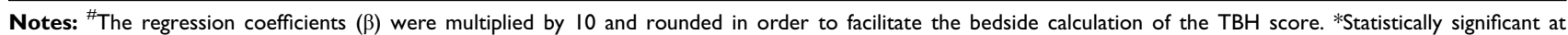
alpha $=0.05$.

Abbreviations: TG, triglyceride; BMI, body mass index.

Table 4 The percentage of CISLD and 95\% confident interval of the low, intermediate and high-risk group for internal and external datasets

\begin{tabular}{|l|l|l|l|}
\hline \multirow{2}{*}{ Groups } & \multicolumn{3}{|l|}{ CISLD, \% (95\% confident interval) } \\
\cline { 2 - 4 } & Internal-training set & Internal-validation set & External set \\
\hline Low & $1.40(1.27,1.53)$ & $1.42(1.25,1.60)$ & $0.87(0.64,1.10)$ \\
Intermediate & $16.23(15.79,16.67)$ & $15.87(15.24,16.49)$ & $15.40(14.43,16.38)$ \\
High & $44.07(43.05,45.09)$ & $44.09(42.57,45.62)$ & $51.63(44.50,58.75)$ \\
\hline
\end{tabular}

Abbreviation: CISLD, chemotherapy induced severe liver damage.

\section{Clinical significance of the TBH score}

From January 1, 2017 to June 30, 2017, 486 patients in these two centers with the same inclusion criteria were prospectively enrolled. Among these patients, 302 patients routinely received one or two kinds of protective medications for liver function to prevent CISLD (prevention group), and the other 184 patients did not receive medications (nonprevention group). There were $175,96,31$ patients with TBH scores $\leq 16$ points, 16 to $\leq 39$ points, and $>39$ points in the prevention group, respectively. The corresponding numbers were 104, 64, and 16 patients in the nonprevention group. Polyene phosphatidylcholine and glutathione were used in the prospective study to prevent CISLD. On the censored day of the study on January 31,2018 , the CISLD rates were $2.5 \%$, $8.9 \%$, and $15.6 \%$ in the prevention group, and the corresponding figures were $4.3 \%, 17.5 \%$, and $55.8 \%$ in the nonprevention group. There were significant differences in the CISLD rates between these two groups for patients with TBH scores of 16 to $\leq 39$ points ( $8.9 \%$ vs $17.5 \%, p=0.042)$ and $>39$ points $(15.6 \%$ vs $55.8 \%, p=0.043)$.

\section{Discussion}

This study explored the baseline characteristics prior to the first administration of chemotherapy of 3870 cancer patients admitted to our hospital in relation to the risk of developing CISLD. Using 2580 patients as a training cohort, we developed a score system to estimate the risk of CISLD using information available about the patients prior to their first cycle of chemotherapy. The score system was validated both internally and externally. In our study, there was a nearly $10 \%$ chance of developing severe liver damage, both in the training cohort and in the validation cohorts. The results showed that some patients with certain characteristics had a very high risk of developing CISLD regardless of the regimen. For the first time, our study presents this prediction model and risk stratification score, which can be used to identify medium-to-high-risk groups of people who may develop CISLD prior to the first cycle of chemotherapy so that certain preventative interventions, modifications of the treatment agents or enhanced monitoring of liver function can be conducted to improve the safety of these patients.

Our score system showed very good accuracy in predicting the possibility of CISLD with a C-index of 0.834 , 0.830 , and 0.817 for the training cohort, internal validation cohort, and external validation cohort, respectively. BMI was found to be the strongest predictor of CISLD among the factors included in the final prediction model. Other studies also showed that high BMI values were closely 
related to liver damage after chemotherapy, but these studies were also limited to patients with liver metastases of colorectal cancer who were undergoing preoperative chemotherapy. ${ }^{14-16}$ In contrast to these studies, the results in our study present that BMI, the strongest predictor of CISLD, is not limited to cancer types, tumor staging, chemotherapy regimens, and number of treatment cycles. In addition to BMI, another factor that influenced the incidence of CISLD is serum TG levels. A previous study suggested that BMI and TG were the most effective predictors of the severity of fatty liver and ultrasound scores in patients with nonalcoholic fatty liver disease (NAFLD). ${ }^{17}$ In addition, high blood pressure may induce fatty liver by triggering insulin resistance. Previous population studies have found that the prevalence of NAFLD in hypertensive patients was twice, ${ }^{18}$ or even three times, higher than that in patients with normal blood pressure. ${ }^{19}$ In animal experiments, it has also been found that hypertension may lead to increased oxidative stress and tissue damage in the liver, thereby affecting the progression of nonalcoholic steatohepatitis ${ }^{20}$ or inducing liver injury and hepatic fibrosis. ${ }^{21,22}$ We can speculate that patients with overweight, hyperlipidemia or hypertension may have mild hepatic steatosis, NAFLD, or liver fibrosis. These preexisting hepatic pathological changes without clinical symptoms or abnormal liver biochemical indicators may be difficult to detect, but they may increase the susceptibility to CISLD.

The main significance of this study is to provide a reference tool for the oncologist's clinical decision-making process. The current dose of chemotherapeutic agents is still mainly based on the body surface area (BSA). However, BSA does not reflect the liver's capacity for metabolic clearance. Our model shows that patients with high BMIs are more susceptible to CISLD and should be treated carefully with reasonable reductions to the chemotherapeutic doses based on a large BSA. In addition, since BMI, TG and hypertension are all related to NAFLD, and patients with NAFLD may poorly tolerate chemotherapy and be prone to CISLD, we may routinely perform liver ultrasounds before chemotherapy to assess whether the patient has mild fatty liver disease that is not usually noticeable. For patients with moderate to high-risk TBH scores, we also have the option of using hepatoprotective drugs prophylactically. In our prospective data of patients assessed as being moderate to high risk, the incidence of CISLD was significantly reduced in those who prophylactically received protective medications for liver function compared to those who did not. Some studies have found that several adjuvant drugs could prevent liver toxicity after chemotherapy, such as tiopronin, ${ }^{23}$ magnesium isoglycyrrhizinate, ${ }^{24}$ S-adenosylmethionine ${ }^{25}$ and even the therapeutic drug bevacizumab. ${ }^{26,27}$ Nonetheless, patients using these drugs should also be closely monitored with liver function tests and follow-up examinations.

There are some limitations in this study. First, the study was a retrospective analysis, not a prospective study. Second, the data of the training group and the validation groups were collected from hospitals from China, which limits the region and race profile of the patients. In addition, we did not analyze the genetic polymorphisms and genotypes of the patients, which may also be related to the risks of CISLD.

\section{Conclusion}

For the first time, we stratified the risks of CISLD by using the $\mathrm{TBH}$ scoring system to easily identify high-risk patients and provided a reliable and operational tool for clinical use to improve the safety of chemotherapy in cancer patients.

\section{Disclosure}

The authors report no conflicts of interest in this work.

\section{References}

1. Lee WM. Drug-induced hepatotoxicity. $N$ Engl J Med. 1995;333 (17):1118-1127. doi:10.1056/NEJM199510263331706

2. Ishak KG, Zimmerman HJ. Morphologic spectrum of drug-induced hepatic disease. Gastroenterol Clin North Am. 1995;24(4):759-786.

3. Chun YS, Laurent A, Maru D, Vauthey JN. Management of chemotherapy-associated hepatotoxicity in colorectal liver metastases. Lancet Oncol. 2009;10(3):278-286. doi:10.1016/S14702045(09)70064-6

4. Carreras E. Veno-occlusive disease of the liver after hemopoietic cell transplantation. Eur J Haematol. 2000;64(5):281-291.

5. Ho VT, Revta C, Richardson PG. Hepatic veno-occlusive disease after hematopoietic stem cell transplantation: update on defibrotide and other current investigational therapies. Bone Marrow Transplant. 2008;41(3):229-237. doi:10.1038/sj.bmt.1705899

6. Brouquet A, Benoist S, Julie C, et al. Risk factors for chemotherapy-associated liver injuries: A multivariate analysis of a group of 146 patients with colorectal metastases. Surgery. 2009;145(4):362-371. doi:10.1016/j.surg.2008.12.002

7. Sorensen P, Edal AL, Madsen EL, Fenger C, Poulsen MR, Petersen OF. Reversible hepatic steatosis in patients treated with interferon alfa-2a and 5-fluorouracil. Cancer. 1995;75(10):2592-2596.

8. Dyson JK, McPherson S, Anstee QM. Non-alcoholic fatty liver disease: non-invasive investigation and risk stratification. $J$ Clin Pathol. 2013;66(12):1033-1045. doi:10.1136/jclinpath-2013-201620

9. van der Bol JM, Mathijssen RH, Loos WJ, et al. Cigarette smoking and irinotecan treatment: pharmacokinetic interaction and effects on neutropenia. J Clin Oncol. 2007;25(19):2719-2726. doi:10.1200/ JCO.2006.09.6115

10. Nakano H, Oussoultzoglou E, Rosso E, et al. Sinusoidal injury increases morbidity after major hepatectomy in patients with colorectal liver metastases receiving preoperative chemotherapy. Ann Surg. 2008;247(1):118-124. doi:10.1097/SLA.0b013e31815774de 
11. Balducci L, Extermann M. Management of cancer in the older person: a practical approach. Oncologist. 2000;5(3):224-237.

12. Chitturi S, Farrell GC. Drug-induced liver disease. Curr Treat Options Gastroenterol. 2000;3(6):457-462.

13. Trotti A, Colevas AD, Setser A, et al. CTCAE v3.0: development of a comprehensive grading system for the adverse effects of cancer treatment. Semin Radiat Oncol. 2003;13(3):176-181. doi:10.1016/ S1053-4296(03)00031-6

14. Makowiec F, Mohrle S, Neeff H, et al.. Chemotherapy, liver injury, and postoperative complications in colorectal liver metastases. $J$ Gastrointest Surg. 2011;15(1):153-164. doi:10.1007/s11605-0101368-7

15. Vauthey JN, Pawlik TM, Ribero D, et al. Chemotherapy regimen predicts steatohepatitis and an increase in 90-day mortality after surgery for hepatic colorectal metastases. J Clin Oncol. 2006;24 (13):2065-2072. doi:10.1200/JCO.2005.05.3074

16. Pawlik TM, Olino K, Gleisner AL, Torbenson M, Schulick R, Choti MA. Preoperative chemotherapy for colorectal liver metastases: impact on hepatic histology and postoperative outcome. $J$ Gastrointest Surg. 2007;11(7):860-868. doi:10.1007/s11605-0070149-4

17. Abangah G, Yousefi A, Asadollahi R, Veisani Y, Rahimifar P, Alizadeh S. Correlation of body mass index and serum parameters with ultrasonographic grade of fatty change in non-alcoholic fatty liver disease. Iran Red Crescent Med J. 2014;16(1):e12669. doi:10.5812/ircmj.12669

18. Bedogni G, Miglioli L, Masutti F, Tiribelli C, Marchesini G, Bellentani S. Prevalence of and risk factors for nonalcoholic fatty liver disease: the dionysos nutrition and liver study. Hepatology. 2005;42(1):44-52. doi:10.1002/hep.20734

19. Donati G, Stagni B, Piscaglia F, et al. Increased prevalence of fatty liver in arterial hypertensive patients with normal liver enzymes: role of insulin resistance. Gut. 2004;53(7):1020-1023. doi:10.1136/ gut.2003.027086
20. Ikuta T, Kanno K, Arihiro K, et al. Spontaneously hypertensive rats develop pronounced hepatic steatosis induced by choline-deficient diet: evidence for hypertension as a potential enhancer in non-alcoholic steatohepatitis. Hepatol Res. 2012;42(3):310-320. doi:10.1111/j.1872-034X.2011.00920.x

21. Hsu CT. Ultrastructural changes in liver damage induced by carbon tetrachloride in spontaneously hypertensive rats and Wistar-Kyoto rats. J Auton Nerv Syst. 1998;70(1-2):79-83.

22. Arima S, Uto H, Ibusuki R, et al. Hypertension exacerbates liver injury and hepatic fibrosis induced by a choline-deficient L-amino acid-defined diet in rats. Int $J$ Mol Med. 2014;33(1):68-76. doi:10.3892/ijmm.2013.1544

23. Li XP, Wen F, Yang W, et al. The role of tiopronin for the prevention of chemotherapy-related liver toxicity in advanced colorectal cancer patients treated with mFOLFOX7: a prospective analysis. Tumori. 2014;100(4):446-451. doi:10.1700/1636.17908

24. Yan Y, Mo Y, Zhang D. [Magnesium isoglycyrrhizinate prevention of chemotherapy-induced liver damage during initial treatment of patients with gastrointestinal tumors]. Zhonghua Gan Zang Bing Za Zhi. 2015;23(3):204-208. doi:10.3760/cma.j.issn.1007-3418.20 15.03.010

25. Santini D, Vincenzi B, Massacesi C, et al. (AdoMet) supplementation for treatment of chemotherapy-induced liver injury. Anticancer Res. 2003;23(6d):5173-5179.

26. Rubbia-Brandt L, Lauwers GY, Wang H, et al. Sinusoidal obstruction syndrome and nodular regenerative hyperplasia are frequent oxaliplatin-associated liver lesions and partially prevented by bevacizumab in patients with hepatic colorectal metastasis. Histopathology. 2010;56(4):430-439. doi:10.1111/ j.1365-2559.2010.03511.x

27. van der Pool AE, Marsman HA, Verheij J, et al. Effect of bevacizumab added preoperatively to oxaliplatin on liver injury and complications after resection of colorectal liver metastases. J Surg Oncol. 2012;106(7):892-897. doi:10.1002/jso.23142 


\section{Supplementary materials}

Table SI Baseline characteristics of patients in the external validation cohort

\begin{tabular}{|c|c|}
\hline Patient's characteristics & Total $(n=4 \mid 3)$ \\
\hline \multicolumn{2}{|l|}{ Background data } \\
\hline Age, yr & $54.3 \pm 12.4$ \\
\hline $\mathrm{BMI}, \mathrm{kg} / \mathrm{m}^{2}$ & $22.0 \pm 3.2$ \\
\hline Gender (male/female) & $254 / 159(61.5 \% / 38.5 \%)$ \\
\hline History of smoking (Yes/No) & $|42 / 27|(34.4 \% / 65.6 \%)$ \\
\hline History of hypertension (Yes/No) & $65 / 348(15.7 \% / 84.3 \%)$ \\
\hline History of CHD (Yes/No) & $15 / 398(3.6 \% / 96.4 \%)$ \\
\hline History of HBV of infection (Yes/No) & $67 / 346(16.2 \% / 83.8 \%)$ \\
\hline History of HCV of infection (Yes/No) & $5 / 408(1.2 \% / 98.8 \%)$ \\
\hline Nucleotide analogue medicine (Yes/No) & $37 / 376(9.0 \% / 91.0 \%)$ \\
\hline History of diabetes (Yes/No) & $33 / 380(8.0 \% / 92.0 \%)$ \\
\hline History of alcoholism (Yes/No) & $66 / 347(16.0 \% / 84.0 \%)$ \\
\hline \multicolumn{2}{|l|}{ Laboratory data } \\
\hline ALB, $g / l$ & $38.7 \pm 5.0$ \\
\hline $\mathrm{BUN}, \mathrm{mmol} / \mathrm{l}$ & $5.2 \pm 5.0$ \\
\hline $\mathrm{Cr}, \mu \mathrm{mol} / \mathrm{l}$ & $69.3 \pm 24.7$ \\
\hline $\mathrm{UA}, \mu \mathrm{mol} / \mathrm{l}$ & $298.8 \pm 97.1$ \\
\hline $\mathrm{GLU}, \mathrm{mmol} / \mathrm{l}$ & $5.7 \pm 4.2$ \\
\hline ALT, U/L & $26.6 \pm 22.9$ \\
\hline AST, U/L & $28.3 \pm 25.0$ \\
\hline ALT/AST & $1.0 \pm 1.6$ \\
\hline $\mathrm{CHOL}, \mathrm{mmol} / \mathrm{l}$ & $4.5 \pm 1.0$ \\
\hline $\mathrm{TG}, \mathrm{mmol} / \mathrm{l}$ & $1.2 \pm 0.8$ \\
\hline CRP, mg/l & $3.9 \pm 9.1$ \\
\hline Cys, mg/l & $0.8 \pm 0.2$ \\
\hline $\mathrm{Hb}, \mathrm{g} / \mathrm{l}$ & $120.6 \pm 23.6$ \\
\hline TBIL, $\mu \mathrm{mol} / \mathrm{l}$ & $13.0 \pm 22.3$ \\
\hline GGT, U/L & $101.0 \pm 145.7$ \\
\hline APTT, s & $28.8 \pm 5.1$ \\
\hline $\mathrm{Fbg}, \mathrm{g} / \mathrm{l}$ & $4.4 \pm 1.5$ \\
\hline INR & $0.9 \pm 0.1$ \\
\hline \multicolumn{2}{|l|}{ Other data } \\
\hline \multicolumn{2}{|l|}{ Tumor type } \\
\hline Gastrointestinal & 146 (35.4\%) \\
\hline Breast & $25(6.1 \%)$ \\
\hline Head/neck & $37(9.0 \%)$ \\
\hline Lung & I 37 (33.2\%) \\
\hline Urinary tract & $9(2.2 \%)$ \\
\hline Gynecologic & 40 (9.7\%) \\
\hline Hematologic & 7 (1.7\%) \\
\hline Others & $12(2.9 \%)$ \\
\hline Liver metastasis (Yes/No) & $41(9.9 \%) / 372(90.1 \%)$ \\
\hline
\end{tabular}

(Continued)
Table SI (Continued).

\begin{tabular}{|l|l|}
\hline Patient's characteristics & Total (n=4I3) \\
\hline Tumor staging & 71 (I7.2\%) \\
\hline II & $118(28.6 \%)$ \\
III & $224(54.2 \%)$ \\
\hline Cycle of chemotherapy & \\
\hline$\leq 2$ & $343(83.1 \%)$ \\
3 or 4 & $70(16.9 \%)$ \\
$>4$ & $0(0)$ \\
\hline Chemotherapeutics & \\
\hline Oxaliplatin (Yes/No) & $73 / 340(16.9 \% / 83.1 \%)$ \\
Irinotecan (Yes/No) & $17 / 396(4.1 \% / 95.9 \%)$ \\
Gemcitabine (Yes/No) & $38 / 375(9.2 \% / 90.8 \%)$ \\
Platinum (Yes/No) & $108 / 305(26.2 \% / 73.8 \%)$ \\
Fluorouracil (Yes/No) & $51 / 362(12.3 \% / 87.7 \%)$ \\
Doxorubicin (Yes/No) & $50 / 363(12.0 \% / 88.0 \%)$ \\
Paclitaxel (Yes/No) & $42 / 37$ (I0.2\%/89.8\%) \\
Docetaxel (Yes/No) & $47 / 366(11.3 \% / 88.7 \%)$ \\
Pemetrexed (Yes/No) & $41 / 372(9.9 \% / 90.1 \%)$ \\
\hline
\end{tabular}

Note: Data are presented as $n(\%)$, mean \pm SD.

Abbreviations: $\mathrm{BMI}$, body mass index; $\mathrm{CHD}$, coronary heart disease; ALB, albumin; BUN, urea nitrogen; $\mathrm{Cr}$, creatinine; UA, uric acid; GLU, blood glucose; ALT, alanine aminotransferase; AST, aspartate aminotransferase; ALT/AST, ratio of alanine aminotransferase to aspartate aminotransferase; $\mathrm{CHOL}$, cholesterol; TG, triglyceride; CRP, C-reactive protein; Cys, cystatin C; Hb, hemoglobin; TBIL, total bilirubin; GGT, gamma-glutamyl transpeptidase; APTT, activated partial thromboplastin time; Fbg, fibrinogen; INR, international normalized ratio.

Table S2 Chemotherapy-induced severe liver damage in the training and internal validation cohort

\begin{tabular}{|l|l|l|l|}
\hline Variables & $\begin{array}{l}\text { Total } \\
(\mathbf{n = 3 8 7 0 )}\end{array}$ & $\begin{array}{l}\text { Training } \\
\text { cohort } \\
(\mathbf{n = 2 5 8 0 )}\end{array}$ & $\begin{array}{l}\text { Internal vali- } \\
\text { dation } \\
\text { cohort } \\
\text { (n=I 290) }\end{array}$ \\
\hline ALT increased & 210 & 146 & 64 \\
AST increased & 273 & 178 & 94 \\
ALP increased & 26 & 18 & 8 \\
Bilirubin increased & 33 & 21 & 12 \\
GGT increased & 41 & 26 & 15 \\
Ascites & 22 & 14 & 8 \\
APTT prolonged & 20 & 14 & 6 \\
Fibrinogen & 20 & 11 & 9 \\
decreased & & 12 & 9 \\
INR increased & 21 & 440 & 225 \\
Total & 666 & & \\
\hline
\end{tabular}

Abbreviations: ALT, alanine aminotransferase; AST, aspartate aminotransferase; ALP, alkaline phosphatase; APTT, activated partial thromboplastin time; GGT, gamma-glutamyl transpeptidase. 
Table S3 Chemotherapy-induced severe liver damage in the external validation cohort

\begin{tabular}{|l|l|}
\hline Variables & Training cohort $(\mathbf{n}=\mathbf{4}$ I 3) \\
\hline ALT increased & 21 \\
AST increased & 25 \\
ALP increased & 3 \\
Bilirubin increased & 2 \\
GGT increased & 4 \\
Ascites & 1 \\
APTT prolonged & 2 \\
Fibrinogen decreased & 1 \\
INR increased & 1 \\
Total & 60 \\
\hline
\end{tabular}

Abbreviations: ALT, alanine aminotransferase; AST, aspartate aminotransferase; ALP, alkaline phosphatase; APTT, activated partial thromboplastin time; GGT, gamma-glutamyl transpeptidase.

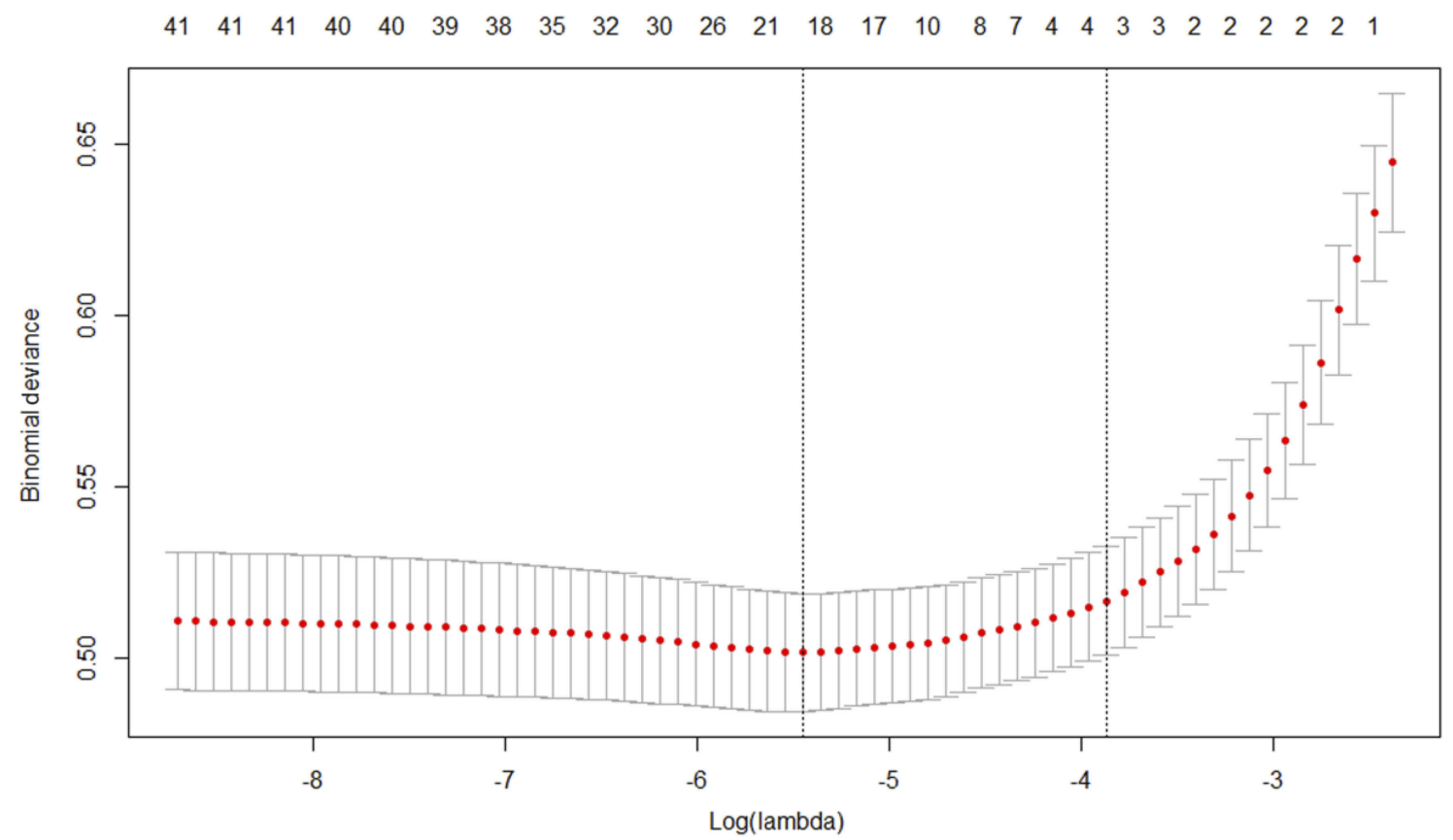

Figure SI Features selection using the LASSO regression. Tuning parameter $(\lambda)$ selection in the LASSO logistic model. The minimum criteria for tenfold cross validation were applied to $\lambda$ selection. Using the I standard error (I-SE) criteria and the minimum criteria, the optimal values of the LASSO tuning parameter ( $\lambda$ ) are indicated by the dotted vertical lines, and a value $\lambda$ of 0.025 was chosen. 


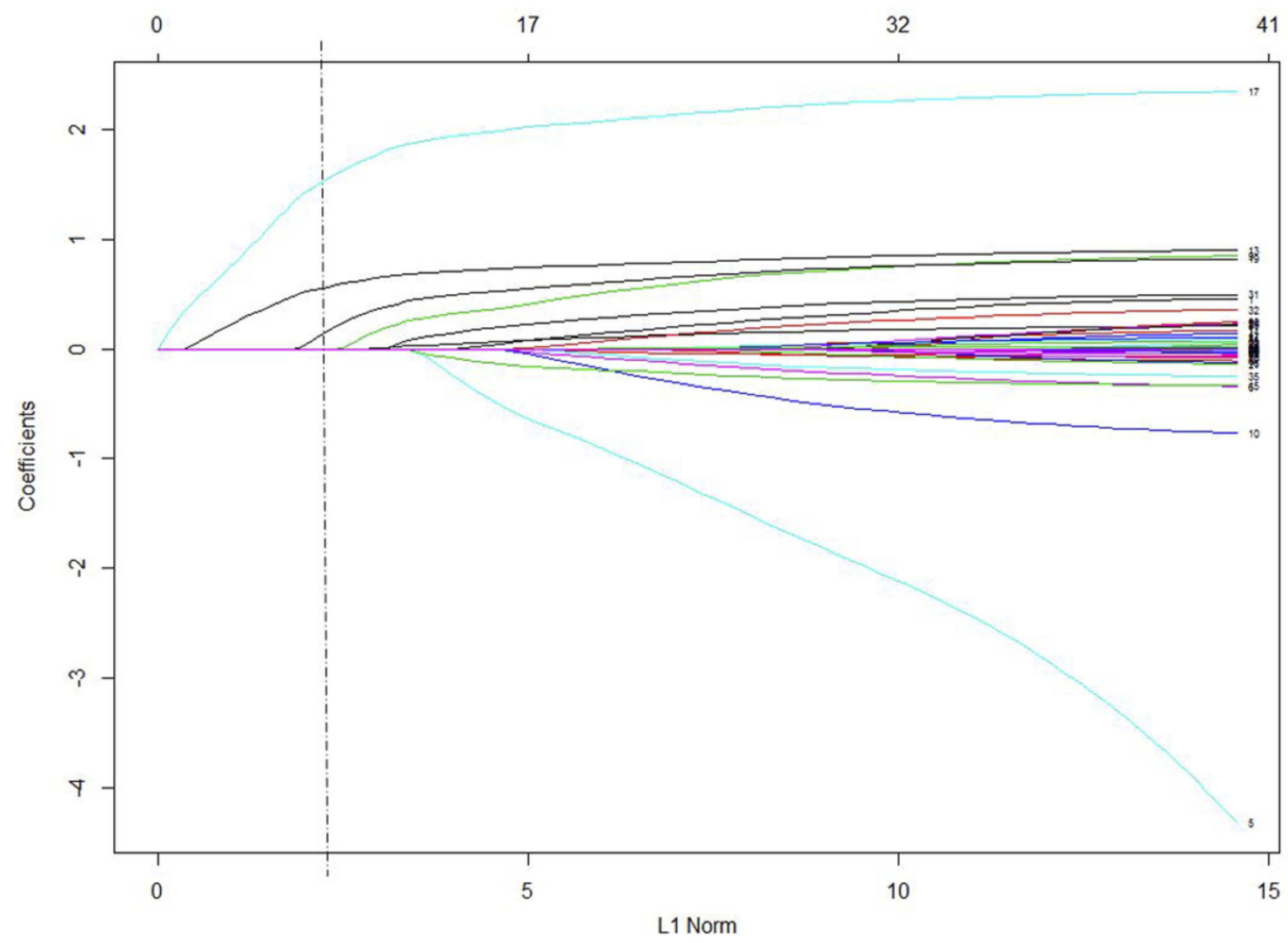

Figure S2 Features selection using the LASSO regression. The LASSO coefficients of the 42 features. A coefficient profile plot was generated against the LI Norm sequence. Vertical line was drawn at the value selected using 10 -fold cross-validation, where optimal $\lambda$ resulted in 8 nonzero coefficients. 


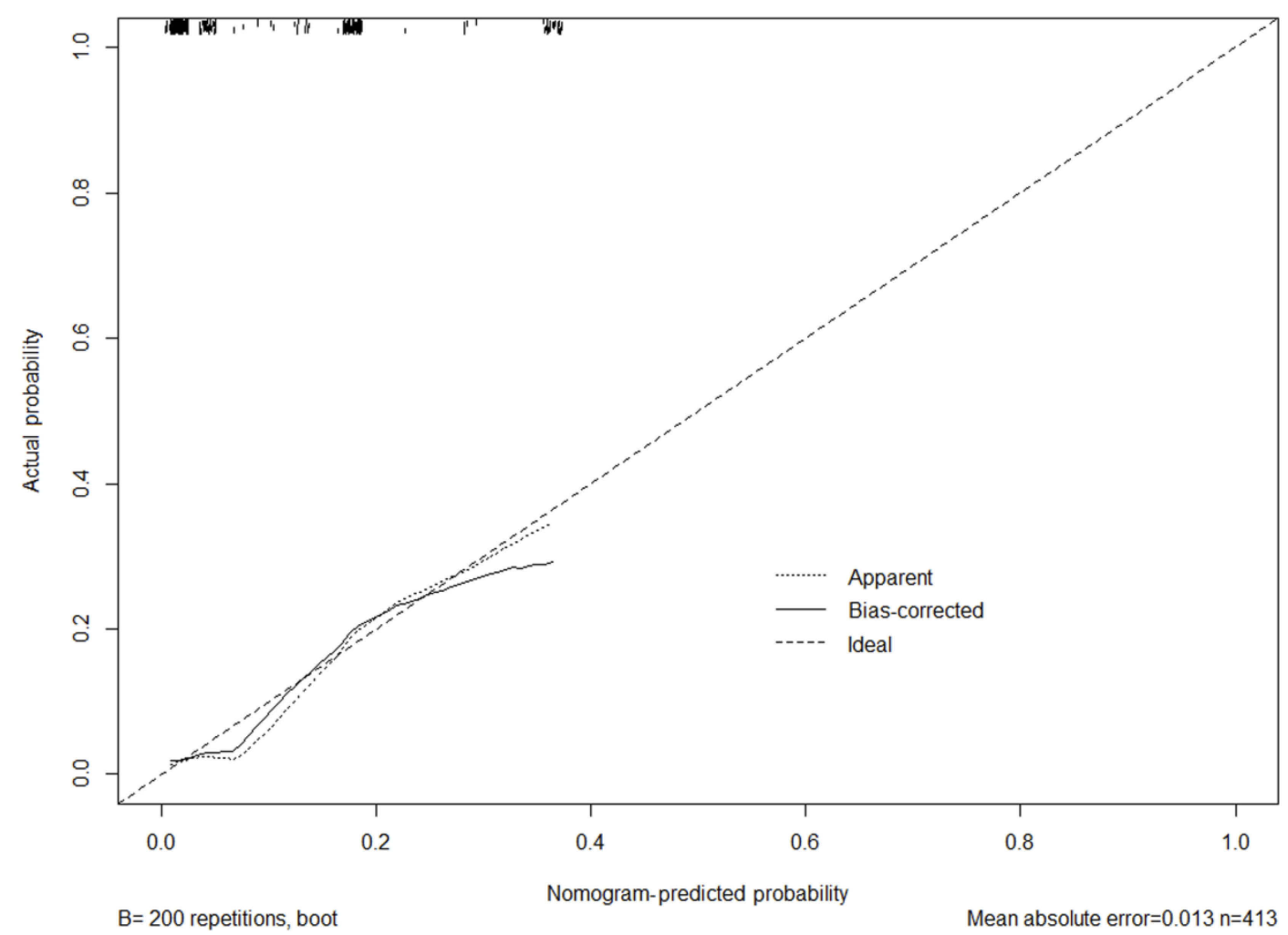

Figure S3 The calibration plot for risk of hepatic function damage in the external validation cohort.

\section{Publish your work in this journal}

Cancer Management and Research is an international, peer-reviewed open access journal focusing on cancer research and the optimal use of preventative and integrated treatment interventions to achieve improved outcomes, enhanced survival and quality of life for the cancer patient.

The manuscript management system is completely online and includes a very quick and fair peer-review system, which is all easy to use. Visit http://www.dovepress.com/testimonials.php to read real quotes from published authors. 